\title{
Topological Quantum Interplay between Magnetic Flux and Mass in an Electron
}

\author{
Vincenzo Vinciguerra* \\ STMicroelectronics, Stradale Primosole 50, 95121, Catania, Italy. \\ *corresponding author email: vincenzo.vinciguerra@st.com
}

\begin{abstract}
A topological quantum interplay between the magnetic flux $\Phi$ and the mass has been investigated, for the case of an electron, by evaluating a gauge-invariant phase factor (a Wilson loop) linked to the electromagnetic gauge field $A_{\mu}$ of the particle. In particular, from this phase factor and the quantization of the magnetic flux variations, a relationship between the mass at rest of the electron $m_{e}$ and its self-energy $\delta m$, arising from radiative corrections, has been obtained also within a QED approach. Besides, a formulation of an energy scale comparable to the energy at rest of an electron-positron pair is proposed. Remarkably, a reckoning of the Bohr's energy of a $W^{+} W^{-}$pair is compatible with constants and parameters usually employed within the electroweak theory and comparable to the energy at rest of an $e^{-} e^{+}$pair.
\end{abstract}

Keywords: electron, topology, magnetic flux quantization, charge, mass, electroweak theory, Bohr's energy, $W$ boson vector.

PACS numbers: Relativistic electron and positron, 41.75.Ht, Topology, 02.40.Pc, Electroweak interactions, 12.15.y, Atomic spectra, 32.30.-r, W bosons, 14.70.Fm.

\section{Introduction}

Of all the massive elementary particles [1] investigated in the standard model (SM) [2], electrons have been definitely central in the development of the semiconductor industry [3], being their properties, either classical or quantal, widely exploited in the whole set of integrated circuits made of downscaled solid state devices.

For technologists and experts involved in the investigation of matter, electrons are fundamental particles upon which devising explanations regarding atoms, molecules, condensed matter and general observed phenomena. Indeed, thanks also to the rise and reliability of computational methods, tested over the years and established on fundamental principles, scientists can implement today a set of theoretical frameworks to further understand the structure of matter. Examples are the ab-initio methods such as tight binding, Hartree Fock (HF) and self-consistent HF $[4,5]$ ), as well as first principles methods, built on the theory of density functional (DFT) [69]. Their use has definitely had an impact on our understanding of quantum effects in solid state thin films (e.g. in the quantum integer and fractional Hall effect) [10] and in the development of new device technologies [11], too.

To complete the picture, the physical foundations of the methods illustrated above rely upon the theory of quantum electrodynamics (QED) [12-13] and its later developments within the 
electroweak theory; making QED the most successful theory in predicting and describing the interaction of electrons with light in every aspect.

However, after more than a century from the discovery [14] of the electron as a fundamental building block of matter, the electron is still perceived mostly as an enigmatic particle in its essence. Indeed, the words of the scholar Wilczeck [15] confirms such a picture: "An electron is a particle and a wave; it is ideally simple, and unimaginably complex; it is precisely understood, and utterly mysterious; it is rigid, and subject to creative disassembly. No single answer does justice to reality".

Recently topology [16] is receiving considerable attention by condensed matter's physicists focusing on the properties of topological phases [17-18]. Nonetheless, topological arguments have been already introduced in physics in the past [19-23]. Indeed, the charge quantization conditions determined by the existence of magnetic monopoles [24, 25], the Arhanov-Bohm effect [26] and the Berry's phase [27] are just acknowledged examples of topological properties arising in specific physical systems.

In general, topology gives us insights on how different parts of a structure are linked together, or can be connected by paths. The Königsberg's bridge problem of Euler [28], the network of covalent bonds of atoms in a molecule [29], the structure of a LAN or a WAN [30] are also examples of topological structures that can occur in space configurations. More specifically, in geometry, surface topology investigates how paths (open or in loops) can be deformed equivalently and continuously without tears or cuts of the surface or, in a similar manner, how surfaces can be modified smoothly into one another (e.g. a cup into a donut!).

Topological aspects determining the quantization properties of the intrinsic magnetic flux of electrons and the significance of the magnetic flux quantization is acknowledged in the field of condensed matter [31-33] as determinant to understand the physics of superconducting state and proved experimentally by Deaver and Fairbank [34], as well as by Doll and Näbauer [35]. Later the role played by the quantization of magnetic flux was extended to the Quantum Hall effect $[36,37]$.

In this work, we investigate the role that a quantization of the intrinsic magnetic flux in a particle plays in determining its properties. In particular, we discuss the topological interplay occurring between the intrinsic magnetic flux of a charged lepton, namely an electron, and its mass.

According to the literature, the topic has been investigated to some extent in the past and for a decade by Jehle [38-43]. Indeed, in Jehle's works, it is reported about the possibility to formulate a theory of the electromagnetic field of charged leptons as resulting from quantized magnetic flux. Jehle pointed out that the magnetic flux is an option that electrodynamics provides to investigate the nature of charged leptons and proved that the Coulomb potential can be obtained as arising from the quantization of the magnetic flux [39]. Although the formulated model was phenomenological and in contradiction with the standard formulation of QED, because of the electromagnetic origin of the mass of the electron, his formulation was an embryonal case of a topological property associated with the magnetic flux of the electron. 
More recently, Saglam [44] and Stein [45] proved that the magnetic flux of an electron in a hydrogen atom is also quantized and that the electron spin contributes to the quantized flux according to $-2 \pi c\langle\hat{s}\rangle / e$, where $\langle\hat{s}\rangle$ is the expectation value of the spin of the particle, $-e$ is its electric charge and $c$ the speed of light. At the best of the knowledge of the author, no further extensive investigations have been reported so far on the topic.

The paper is organized as follows. First, the topological quantum interplay between the magnetic flux $\Phi$ and the mass, for the case of an electron, is investigated by evaluating a gauge-invariant phase factor associated with the Wilson loop of the electromagnetic field $A_{\mu}$ generated by the particle. In particular, from the phase factor and the quantization of the magnetic flux variations, a relationship between the mass at rest of the electron $m_{0}$ and its self-energy $\delta m$ is obtained also within a QED approach. Hence, the formulation of an energy scale comparable with the energy at rest of an electron and compatible with constants and parameters usually employed within the electroweak theory is discussed. Finally the conclusions are reported.

\section{Topological interplay between magnetic flux and mass}

Whenever a particle of charge $q$ undergoes a space-time loop $C$, in the gauge field $A^{\mu}$ generated by an electron, it is possible to evaluate a gauge-invariant quantity [46]

$$
e^{\frac{i q}{\hbar c} \oint_{C} \mathrm{~A}_{\mu} d x^{\mu}}|\psi\rangle=e^{i q \alpha}|\psi\rangle
$$

that determines a phase shift $q \alpha$ of the state $|\psi\rangle$. From the derivative of eq. 1 with respect to $q$, it results that the quantity $\frac{q}{\hbar c}\left\langle\oint_{C} \mathrm{~A}_{\mu} d x^{\mu}\right\rangle$ determines the phase $q \alpha$ :

$$
\frac{q}{\hbar c}\left\langle\oint_{C} \mathrm{~A}_{\mu} d x^{\mu}\right\rangle=q \alpha
$$

On the other hand, the gauge-invariant quantity on the first member of eq. 2 can be evaluated independently. For example, for the case of an electron at rest with spin up, in a semi-classical approach, it reads as:

$$
\left\langle\oint_{C} \quad \mathrm{~A}_{\mu} d x^{\mu}\right\rangle=c \frac{-e\langle\varphi(\vec{r}, t)\rangle}{-e} T-\Phi(\uparrow)=\frac{2 c}{-e} \delta m_{e s} c^{2} T-\Phi(\uparrow)
$$

Where $\langle\varphi(\vec{r}, t)\rangle$ is the expectation value of the time-component of the 4-vector potential $A^{\mu}$, $\delta m_{e s} c^{2}$ works as an electrostatic energy and the period $T$ is a characteristic time of the electron, which hereafter will be considered equal to $T=\frac{\pi \hbar}{m_{e} c^{2}}$.

In order to calculate the magnetic flux of an electron we proceed by considering the line integral of the operator $\vec{A}$ on a closed circular loop $C_{r}=\{r(t) \mid t=0 \rightarrow T\}$ in the space-coordinates and average it over all possible values:

$$
\Phi=\left\langle\oint_{C_{r}} \vec{A} \cdot d \vec{r}\right\rangle=\int d V \oint_{C_{r}} \psi^{\dagger}(r, t) \vec{A} \psi(r, t) \cdot d \vec{r}=\int d V^{\prime} \oint_{C_{r^{\prime}}} \psi^{\dagger}\left(r^{\prime}, t^{\prime}\right) \vec{A} \psi\left(r^{\prime}, t^{\prime}\right) \cdot d \overrightarrow{r^{\prime}}
$$


In doing such a calculation, we can renominate $r$ in $r^{\prime}$, as we expect that the variables $r$ and $r^{\prime}$ play a symmetric role.

In the semi-classical approach that we are following, according to eq. 4 the magnetic flux of an electron reads as:

$$
\Phi=\left\langle\oint_{C_{r}} \vec{A} \cdot d \vec{r}\right\rangle=\frac{1}{c}\left\langle\int \oint_{C_{r}} \frac{\vec{j} \cdot d \vec{r}}{\left|\vec{r}-r^{\prime}\right|} d V^{\prime}\right\rangle
$$

In particular, for the case of an electron at rest, the magnetic moment $m_{z}$ of an electron depends only on the spin, and it's an observable that commutes with the Dirac's Hamiltonian. It can be shown that:

$$
\Phi=\frac{1}{c}\left\langle\int \oint_{C_{r}} \frac{\vec{j} \cdot d \vec{r}}{\left|\vec{r}-\overrightarrow{r^{\prime}}\right|} d V^{\prime}\right\rangle=\frac{4 \pi}{c}\left\langle\int \frac{1}{2} \frac{(\vec{r} \times \vec{j})_{z}}{\left|\vec{r}-\overrightarrow{r^{\prime}}\right|} d V^{\prime}\right\rangle=\frac{4 \pi}{c}\left\langle\int \frac{m_{z} \psi^{\dagger}\left(r^{\prime}, t^{\prime}\right) \psi\left(r^{\prime}, t^{\prime}\right)}{\left|\vec{r}-\overrightarrow{r^{\prime}}\right|} d V^{\prime}\right\rangle
$$

By reasoning on the quantized value that the magnetic moment $m_{z}$ of an electron at rest assumes for a state with spin up, we can deduce that:

$$
\Phi(\uparrow)=-\frac{4 \pi \hbar c}{2 e m_{e} c^{2}}\left\langle\int \frac{e^{2} \psi^{\dagger}\left(r^{\prime}, t^{\prime}\right) \psi\left(r^{\prime}, t^{\prime}\right)}{|\vec{r}-\vec{r}|} d V^{\prime}\right\rangle .
$$

Hence the gauge-invariant quantity in eq. 2, can be evaluated as

$$
\left\langle\oint_{C} \mathrm{~A}_{\mu} d x^{\mu}\right\rangle=\frac{\delta m_{e s}}{m_{e}} \frac{2 \pi \hbar c}{e}
$$

In the event, the phase in eq. 2 amounts to:

$$
\frac{q}{\hbar c}\left\langle\oint_{C} \mathrm{~A}_{\mu} d x^{\mu}\right\rangle=\frac{q}{\hbar c} \frac{\delta m_{e s}}{m_{e}} \frac{2 \pi \hbar c}{e}=\frac{\delta m_{e s}}{m_{e}} \frac{2 \pi q}{e} .
$$

Moreover, in the hypothesis that there's a certain arbitrariness in the choice of the charge $q$, we can choose it such that the gauge-invariant quantity in eq. 3 reads as:

$$
\frac{q}{\hbar c}\left\langle\oint_{C} \mathrm{~A}_{\mu} d x^{\mu}\right\rangle=2 \pi n, \text { with } n \in \mathbb{Z}
$$

If this holds, the ratio between $\delta m_{e s}$ and $m_{e}$, must satisfy the equation:

$$
\frac{\delta m_{e s}}{m_{e}}=\frac{n}{q / e}
$$

On the other hand, also the magnetic flux is quantized and equals 


$$
\Phi(\uparrow)=-\frac{4 \pi \hbar c}{e} \frac{\delta m_{e s}}{m_{e}}=-\frac{4 \pi \hbar c}{e} \frac{n}{q / e},
$$

for the case of an electron at rest with spin up, whereas, for an electron at rest with spin down the magnetic flux reads

$$
\Phi(\downarrow)=\frac{4 \pi \hbar c}{e} \frac{\delta m_{e s}}{m_{e}}=\frac{4 \pi \hbar c}{e} \frac{n}{q / e} .
$$

By imposing that the variation of the magnetic flux from spin down to spin up is equal to the quantum of the magnetic flux:

$$
\Phi(\downarrow)-\Phi(\uparrow)=\frac{8 \pi \hbar c}{e} \frac{n}{\frac{q}{e}}=\frac{2 \pi \hbar c}{e}
$$

we obtain that the charge $q$ must satisfy the condition $q=4 n e$.

Moreover, by defining $\delta m_{e m} c^{2}=4 \delta m_{e s} c^{2}$ as an electromagnetic energy linked to the electron, the magnetic flux can be expressed as:

$$
\Phi(\uparrow)=-\frac{\pi \hbar c}{e} \frac{\delta m_{e m}}{m_{e}} .
$$

From classical arguments it's reasonable to expect such a dependence. In fact, the magnetic flux $\Phi(\uparrow)$ can be expressed in terms of the magnetic moment of the electron $-\mu_{B}$, where $\mu_{B}$ is the Bohr magneton, as:

$$
\Phi(\uparrow)=-\frac{1}{2} \frac{2 \pi \hbar c}{e} \frac{\delta m_{e m}}{m_{e}}=-\frac{e}{m_{e} c} \frac{\hbar}{2} \frac{2 \pi \delta m_{e m} c^{2}}{e^{2}}=-\mu_{B} \frac{2 \pi \delta m_{e m} c^{2}}{e^{2}} .
$$

Indeed, the magnetic dipole moment $\mu$ determined by a current $i$ flowing, e.g. in a circular coil of radius $r$, is $\mu=i \pi r^{2}$. The magnetic field generated at the center of the coil is $B=\frac{2 \pi i}{r}$, and the magnetic flux is approximately $\Phi=B \pi r^{2}$. By combining previous equations, we can express the magnetic flux in terms of the magnetic moment $\mu$. In fact, $\Phi=B \pi r^{2}=\frac{2 \pi i}{r} \pi r^{2}=\mu \frac{2 \pi}{r}$. A comparison with equation (16) provides the value $\frac{1}{r}=\frac{\delta m_{e m} c^{2}}{e^{2}}$.

By considering also possible QED corrections for the magnetic moment and indicating with $\delta m_{e s} c^{2}$ the contribution of the electrostatic energy, eq. 7 reads as:

$$
\Phi(\uparrow)=-\frac{g_{s}}{2} \frac{8 \pi \hbar c}{2 e m_{e} c^{2}} \delta m_{e s} c^{2},
$$

where $g_{s}$ is the spin g-factor of the electron.

In conclusion, the gauge-invariant quantity appearing in eq. (2), can be expressed as:

$$
\frac{e}{\hbar c}\left\langle\oint_{C} \mathrm{~A}_{\mu} d x^{\mu}\right\rangle=2 \pi \frac{g_{s}}{2} \frac{\delta m_{e m}}{m_{e}}=2 \pi,
$$


and being quantized in units of $2 \pi$, it implies that there must exist an interplay between the magnetic flux and the mass, such that:

$$
\frac{g_{s}}{2} \frac{\delta m_{e m}}{m_{e}}=1
$$

Clearly such a conclusion has been achieved by pursuing a semi-classical approach. In fact, within the QED formulation it is not possible to evaluate a quantity such as the electrostatic selfenergy of the electron. However, from the gauge-invariant quantity appearing in eq. (2), we can consider that $d x^{\mu}=\gamma^{\mu} c d \tau$, where $\gamma^{\mu}$ are the Dirac Matrices and $\tau$ is an intrinsic time, characteristic of the system. If this is the case, by evaluating the line integral as an integral over the time $\tau$, and the expectation value turns out the value of a quantity which has the dimension of an energy $e\left\langle\mathrm{~A}_{\mu} \gamma^{\mu}\right\rangle$. On the other hand, within the QED formulation the self-interaction of the electron with its own electromagnetic field is determined as the self-energy $\delta m c^{2}$ of the electron. Consequently, the gauge-invariant quantity of eq (2) can be estimated as

$$
\frac{e}{\hbar c}\left\langle\oint_{C} \mathrm{~A}_{\mu} d x^{\mu}\right\rangle=\frac{e}{\hbar c}\left\langle\int_{0}^{\tau} \mathrm{A}_{\mu} \frac{d x^{\mu}}{d c \tau} c d \tau\right\rangle=\frac{e}{\hbar c} c \tau\left\langle\mathrm{A}_{\mu} \gamma^{\mu}\right\rangle=\frac{\tau}{\hbar}\left\langle\mathrm{A}_{\mu} e \gamma^{\mu}\right\rangle=\frac{\tau}{\hbar} \delta m c^{2}
$$

where the characteristic time $\tau$ is set as $\tau=\frac{g_{s}}{2} \frac{2 \pi \hbar}{m_{e} c^{2}}$, so that it can be related to the magnetic moment of the electron. In fact,

$$
\Phi(\uparrow)=-\mu_{B} \frac{2 \pi \delta m c^{2}}{e^{2}}=-\frac{g_{s}}{2} \frac{e}{m_{e} c} \frac{\hbar}{2} \frac{2 \pi \delta m c^{2}}{e^{2}}=-\frac{1}{m_{e}} \frac{\hbar}{2} \frac{2 \pi \delta m c}{e},
$$

and the variation of the magnetic flux provides the value

$$
\Phi(\downarrow)-\Phi(\uparrow)=\frac{g_{s}}{2} \frac{2}{m_{e}} \frac{\hbar}{2} \frac{2 \pi \delta m}{e}=\frac{g_{s}}{2} \frac{\hbar c}{e} \frac{2 \pi \delta m}{m_{e}}=\frac{c}{e} \frac{g_{s}}{2} \frac{2 \pi \hbar \delta m c^{2}}{m_{e} c^{2}}=\frac{c \tau}{e} \delta m c^{2} .
$$

In conclusion, the Wilson loop appearing in eq. (2) is equal to 1 and reads as

$$
\left\langle\psi\left|e^{\frac{i e}{\hbar c} \oint_{C} \mathrm{~A}_{\mu} d x^{\mu}}\right| \psi\right\rangle=e^{i \frac{\tau}{\hbar} \delta m c^{2}}=e^{i 2 \pi \frac{g_{S} \delta m}{2 m_{e}}}=1,
$$

if the gauge invariant quantity appearing in eq. 1 is such that:

$$
\frac{g_{s}}{2} \frac{\delta m}{m_{e}}=1
$$

In table I, we summarize such considerations by evaluating the normalized magnetic flux $\Phi_{\text {norm }}=$ $-\mu_{B} \frac{2 \pi \delta m_{E M} c^{2}}{e^{2}} / \frac{2 \pi \hbar c}{e}$ as determined by the confinement size or scale length, for the cases of two representative spin $1 / 2$ charged particles (antiparticles), namely the electron (positron) and the proton (antiproton). The case of the electron is reported for the Bohr radius, the classical radius of the electron, the Compton wavelength and is compared with the calculation obtained from the evaluation of the radiative corrections of the electron mass at first order as obtained from the QED theory. The case of the electron, considered as a point and structure-less particle, is compared with the case of the proton, which is different, since its size is determined by the confinement of its constituent quarks. 


\begin{tabular}{|c|c|c|c|}
\hline Particle (antiparticle) of spin $1 / 2$ & \multicolumn{2}{|c|}{ Confinement size (or scale length) } & Normalized magnetic flux $\Phi_{\text {norm }}=$ \\
\hline \multirow[t]{5}{*}{ Electron (positron) } & Bohr radius & $a_{0}=\hbar / m_{e} c \alpha$ & $-\frac{e}{m_{e} c} \frac{\hbar}{2} \frac{2 \pi m_{e} c \alpha}{\hbar} / \frac{2 \pi \hbar c}{e}=-\frac{1}{2} \alpha^{2}$. \\
\hline & Classical radius & $r_{0}=e^{2} / m_{e} c^{2}$ & $-\frac{e}{m_{e} c} \frac{\hbar}{2} \frac{2 \pi m_{e} c^{2}}{e^{2}} / \frac{2 \pi \hbar c}{e}=-\frac{1}{2}$ \\
\hline & Compton wavelength & $\lambda_{0}=\hbar / m_{e} c$ & $-\frac{e}{m_{e} c} \frac{\hbar}{2} \frac{2 \pi m_{e} c}{\hbar} / \frac{2 \pi \hbar c}{e}=-\frac{1}{2} \alpha$ \\
\hline & $\begin{array}{l}\text { Determined by the electron self- } \\
\text { energy } \delta m[12]\end{array}$ & $r=\frac{e^{2}}{\delta m}$ & $-\frac{1}{2} \frac{\delta m}{m_{e}}$ \\
\hline & & $\begin{array}{l}\text { Taking into account of the } \\
\text { spin g-factor } g_{s} \text { of the } \\
\text { electron. }\end{array}$ & $\begin{array}{c}-\frac{g_{s}}{2} \frac{2 \delta m_{e s}}{m_{e}}=-\frac{g_{s}}{2} \frac{4 \delta m_{e s}}{2 m_{e}}= \\
-\frac{g_{s}}{2} \frac{\delta m_{e m}}{2 m_{e}}=-\frac{g_{s}}{2} \frac{\delta m}{2 m_{e}}\end{array}$ \\
\hline Proton (antiproton) & Proton radius & $r_{P}=0.8768 \mathrm{fm}[47]$ & $\frac{r_{0}}{r_{P}} \frac{m_{e}}{m_{P}} \approx 10^{-3}$ \\
\hline
\end{tabular}

Table I. Normalized magnetic flux $\Phi_{n o r m}=-\mu_{B} \frac{2 \pi \delta m_{e m} c^{2}}{e^{2}} / \frac{2 \pi \hbar c}{e}$ for different scale lengths or confinement sizes of the spin 1/2 particle. Normalization has been done with respect to the quantum flux $\frac{2 \pi \hbar c}{e}$. For the case of an electron (positron) different scale lengths can be considered. By taking into account the electron spin g-factor, a variation of the normalized magnetic flux $\Delta \Phi_{\text {norm }}$ for an electron flipping from spin up to down is in module equal to $\left|\Delta \Phi_{\text {norm }}\right|=$ $\frac{g_{s}}{2} \frac{4 \delta m_{e s}}{m_{e}}=\frac{g_{s}}{2} \frac{\delta m_{e m}}{m_{e}}=\frac{g_{s}}{2} \frac{\delta m}{m_{e}}$. Such a variation equals unity, if $\delta m_{e m}=m_{e} / \frac{g_{s}}{2}$. Finally for the case of the proton, we consider that the minimum length scale is determined by the radius of the proton, which, being a composite particle, is determined by its constituent quarks.

In Figure 1, from a to d, a set of schematizations is also reported in order to show the evolution from classical models of the electromagnetic field of the electrons to QED model of the selfinteraction of the electron with its own electromagnetic field. In particular, in Fig.1a we report a representation of the lines of force of the electric and magnetic fields that can be deduced from classical electrodynamics considerations [48]. In Fig.1b we report a representation of the main features and properties of an electron at rest, according to quantum mechanics. Along with its charge $-e$ and its mass at rest $m_{e}$, we have to consider the intrinsic angular momentum or spin $1 / 2$ of the particle, up in this specific case, its magnetic moment $-\mu_{B}$ (where $\mu_{B}$ is a Bohr magneton unit) and the associated flux of the magnetic field $\Phi$, which is independent of the specific contour line $C_{r}$. In order to evidence the topology which is behind the quantum mechanical properties of an electron, we report an analogical model of the property of spin rotation which is well represented by the topological properties of a Möbius strip (Fig.1c). Fig. 1d reports the one-loop Feynman diagram of the one-loop electron self-energy according to QED. 


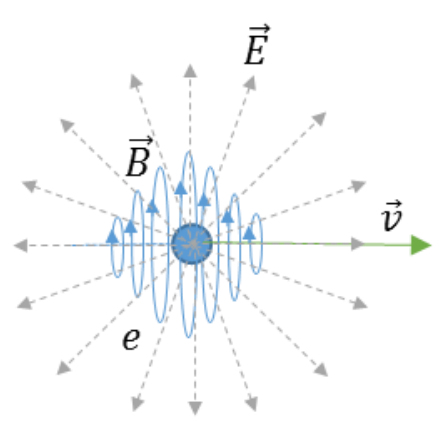

Fig.1a Representation of the lines of force of the electric $\vec{E}$ and magnetic fields $\vec{B}$ as deduced from classical electrodynamics [47] for a moving charged particle, being $\vec{v}$ the vector velocity.

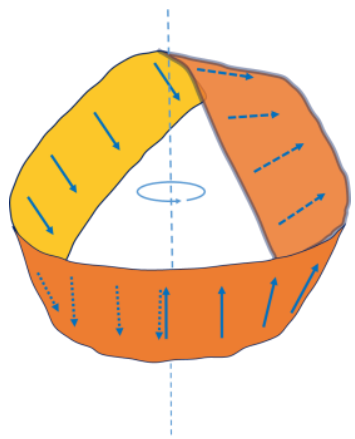

Fig.1c A Möbius strip can work as an analogical model reproducing the topological properties of a spin $1 / 2$ particle subject to a $2 \pi$ rotation. In the specific case, a spin up will turn into a spin down particle, after following the one-sided surface of the strip and rotating of $2 \pi$.

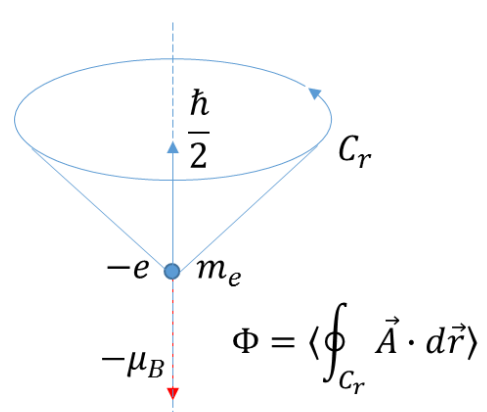

Fig.1b Representation of the main features of an electron at rest. An electron, even at rest, has a magnetic field which determines a magnetic moment of $-\mu_{B}$ (a Bohr magneton), for an electron with spin up. The associated magnetic flux determined by the electron is independent of the line path and is quantized in units of $\Phi(\uparrow)=-\frac{2 \pi c \hbar}{2 e} \frac{\delta m_{e m}}{m_{e}}$.

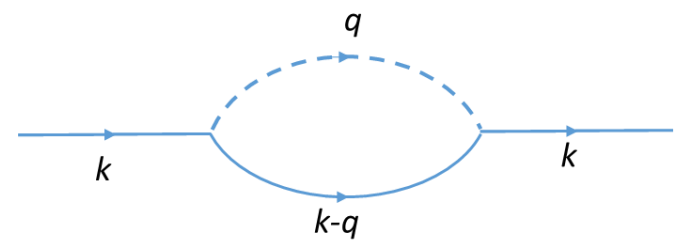

Fig.1d Feynman diagram of the one-loop electron selfenergy. Within the QED formulation the self-interaction of the electron with its own electromagnetic field is determined as the self-energy $\delta m c^{2}$ of the electron. By evaluating the line integral in eq. 2 as an integral over the time $\tau$, the expectation value turns out an expectation value of a quantity which has the dimension of an energy $e\left\langle\mathrm{~A}_{\mu} \gamma^{\mu}\right\rangle$. 


\section{Formulation of an energy scale comparable to the energy at rest of the electron}

In the following an application of equation 19 is considered in order to provide a formulation of an energy scale which is comparable to the energy at rest of the electron. As a matter of fact, radiative corrections within the QED theory provide a contribution to the self-energy determination of the electron [12, 13]. Indeed, by opportunely choosing the cut-off energy required in the QED formulation, a value of the self-energy which is comparable with the energy at rest of the electron can be determined. However, concerning the origin of masses, the diversification of lepton masses can be explained by a Yukawa mechanism of coupling of massless charged leptons with the Higgs field that spontaneously breaks the gauge symmetry [12]. If this is the case we should be able to provide an evaluation of the electron mass within a minimal extension of a scheme which takes into account somehow of the electroweak forces.

In this respect, we may observe as in defining the classical radius $r_{o}$ of the electron, the mass at rest of the electron is proportional to the fine structure constant $\alpha$, whereas the classical radius of the electron can be associated to the Compton wavelength of a more massive particle whose mass reads $\frac{m_{e} c^{2}}{\alpha}=\frac{\hbar c}{r_{0}}$. If we consider the Barut formula [49] $m_{\mu}=m_{e}+m_{e} \frac{3}{2 \alpha}$ for the mass of a muon, being $\frac{m_{e} c^{2}}{\alpha}=\frac{\hbar c}{r_{0}}=\frac{2}{3}\left(m_{\mu}-m_{e}\right) c^{2}$, we can observe as the mass associated to the classical radius of the electron is of the same order of magnitude of the mass of a muon. In such a way we can provide a value of the mass of the electron in terms of that of a muon. However, usually we do the opposite, namely we express the mass of heavier leptons in term of the lightest lepton mass, the electron.

On the other hand, we can conjecture that the dependence of the mass of the electron on the fine structure constant $\alpha$ is reasonable but it may involve a higher power of $\alpha$, such as $\alpha^{2}$. If this is the case, the $\alpha^{2}$ quantity must be multiplied by the mass value of a heavier particle opportunely chosen. Moreover, we can also expect that the unknown equation expressing an energy, should be in a certain way related to standardized expressions of use within the quantum theory among which we can make a choice. In particular, a mass-energy formula that we can consider stems from the atomic theory. In fact, the equation that relates the Bohr energy of the hydrogen energy levels to the mass of the electron has the same dependence on $\alpha^{2}$. Moreover, if we want to pursue the determination of a formula for the mass of the electron within a minimal extension scheme which includes quantities appearing in the electroweak theory, we have to consider the possibility that such heavier mass might be related to the masses of the vector bosons mediating the weak force. Let us devise a thought experiment hence. Namely, let us consider a pair $W^{+} W^{-}$of charged $W$ vector bosons that undergoes a scattering process at very low energy. If this is the case, neglecting the very short lifetime of such particles, it results that the scattering amplitudes determined by the Coulomb scattering are influenced by the possible bound states determined by the $W^{+} W^{-}$system. The energy of such bound states is lowered with respect to $2 M_{W} c^{2}$ by the Bohr energy determined by the electromagnetic interaction of the $W^{+} W^{-}$pair, resulting, as a first approximation, in a binding energy, $E_{B}=\frac{1}{2} \alpha^{2} \frac{M_{W} c^{2}}{2}$, of $1.07 \mathrm{MeV}$. This energy value is quite close to the energy of an $e^{+} e^{-}$pair, which amounts to $2 m_{e} c^{2}=1.02 \mathrm{MeV}$ 
when both particles are at rest. And indeed, we can say that the energy scale of an electron at rest is comparable to half of the Bohr energy of a $W^{+} W^{-}$pair:

$$
m_{e} c^{2} \approx \frac{1}{8} \alpha^{2} M_{W} c^{2}
$$

Moreover, the difference $2 \Delta E$ between the Bohr energy $E_{B}$ and the electron-positron pair mass, results of $48.15 \mathrm{keV}$ :

$$
2 \Delta E=\frac{1}{4} \alpha^{2} M_{W} c^{2}-2 m_{e} c^{2}=48.15 \mathrm{keV} .
$$

Indicating as $\delta M_{W}=2.085 \pm 0.042 \mathrm{GeV}$ [50] the decay width of a charged vector boson, assuming that $2 \delta M_{W}$ is the uncertainty of the mass of the $W^{+} W^{-}$pair, it results that:

$$
\frac{1}{4} \alpha^{2}\left(2 \delta M_{W}\right) c^{2}=55.5 \pm 1.1 \mathrm{keV} .
$$

In the hypothesis that such a difference turns out from an indetermination on the mass of the charged boson vector $W$, we can wright equation (26) as:

$$
\Delta E=\frac{1}{8} \alpha^{2} M_{W} c^{2}-m_{e} c^{2} \approx \frac{1}{8} \alpha^{2} 2 \delta M_{W} c^{2} .
$$

Therefore, the error associated with the mismatch of the two energy scales, the mass of the electron $m_{e} c^{2}$ and the corrected Bohr energy of a $W^{+} W^{-}$pair $\frac{1}{8} \alpha^{2}\left(M_{W}-2 \delta M_{W}\right) c^{2}$ is:

$$
1-\frac{\frac{1}{8} \alpha^{2} M_{W} c^{2}-\frac{1}{8} \alpha^{2} 2 \delta M_{W} c^{2}}{m_{e} c^{2}} \approx 0.7 \%
$$

and differs only by $0.7 \%$ from the mass at rest of an electron.

It is also possible to go further in our evaluation. Indeed, we can introduce an angle $\theta$ such that to take into account of the deviation of difference reported in eq. 26 with respect to the quantity calculated in eq. 27 :

$$
\frac{1}{4} \alpha^{2}\left(2 \delta M_{W}\right) c^{2} \cos \theta=\frac{1}{4} \alpha^{2} M_{W} c^{2}-2 m_{e} c^{2}
$$

It results that a reckoning of $\cos \theta$ provides a value of $\theta$ which amounts to $\theta=$ $\operatorname{acos}\left(\frac{48.15 \mathrm{keV}}{55.5 \pm 1.1 \mathrm{keV}}\right)=(29.8 \pm 2)^{\circ}$. Such a value is comparable with the Weinberg angle $\theta_{W}=$ $28.1^{\circ}$. In the hypothesis that the angle $\theta$ is the Weinberg angle $\theta_{W}$, we can gain a value for the energy scale that we are evaluating which is even closer to the energy at rest of the mass of the electron:

$$
m_{e} c^{2}=\frac{1}{8} \alpha^{2} M_{W} c^{2}-\frac{1}{8} \alpha^{2}\left(2 \delta M_{W}\right) c^{2} \cos \theta_{W}=0.5106(5) \mathrm{MeV}(0.08 \%),
$$

and such a value differs only by $0.08 \%$ with respect to the mass of the electron. On the other hand, the Bohr energy is an electromagnetic form of energy, by considering the results reported in eq. (19), eq. (31) must be multiplied by half of the spin g-factor $\frac{g_{s}}{2}$. By doing such a 
correction, we can obtain a value of an energy scale which differs by only $0.04 \%$ with respect to the mass at rest of the electron:

$$
m_{e} c^{2}=\frac{g_{s}}{2}\left(\frac{1}{8} \alpha^{2} M_{W} c^{2}-\frac{1}{8} \alpha^{2}\left(2 \delta M_{W}\right) c^{2} \cos \theta_{W}\right)=0.5112(5) \mathrm{MeV}(0.04 \%) .
$$

Figures $2 \mathrm{a}$ and $2 \mathrm{~b}$ sum up, in sketches, what described so far.

Is this what the mass of an electron should look like? Does eq. 32 determines the mass of the electron? Although the advantage of such a formulation is the use of parameters and quantities which are proper of the electroweak theory, in spite of the close value of the calculated energy scale in eq. 32 to the energy at rest of the electron, a skeptic reader might also consider that the previous heuristic reasoning leads only to a fortunate guess of an energy value which is very close to the mass of an electron. As a fact, we cannot exclude that such a conclusion is a pure coincidence arising from the calculation of the Bohr energy of a $W^{+} W^{-}$pair. Although of the many clues provided, such as the Bohr energy and its corrections which are very close to the energy at rest of an $e^{+} e^{-}$pair, the Bohr radius of the $W^{+} W^{-}$system which is comparable to the classical electron radius, a critical point to consider is the appropriate identification of the angle $\theta$. However, independently of the possible implications which are behind eq. 25 and 32, it is sure that eq. 25, a Bohr energy, is the value of an energy scale comparable to the energy scale of an electron at rest which has been formulated by means of the mass $M_{W}$ of the $W$ particle.

\section{Conclusions}

In conclusion, by evaluating a gauge-invariant phase factor (that is a Wilson loop) linked to the electromagnetic gauge field $A_{\mu}$ of the particle, namely an electron, a topological quantum interplay between the magnetic flux $\Phi$ and the mass has been demonstrated, In particular, it has been shown that, from the phase factor and the quantization of the magnetic flux variations, a relationship between the mass at rest of the electron $m_{\mathrm{e}}$ and its self-energy $\delta m$, arising from radiative corrections, can be obtained, also within a QED approach. Besides, a formulation of an energy scale comparable to the energy at rest of an electron-positron pair is proposed. It is remarkable that a reckoning of the Bohr energy of a $W^{+} W^{-}$pair, which is compatible with constants and parameters usually employed within the electroweak theory, is comparable to the energy at rest of an $e^{-} e^{+}$pair. Moreover, it has been shown how corrections can be provided such that the calculated energy scale approaches better the value of an $e^{-} e^{+}$pair. However at this stage we cannot exclude that such a conclusion is a pure coincidence arising from our speculation. Nevertheless, independently of such speculations, it is certain that the electron, the lightest lepton, will continue to be exploited in the entire spectrum of technological applications that will emerge. 


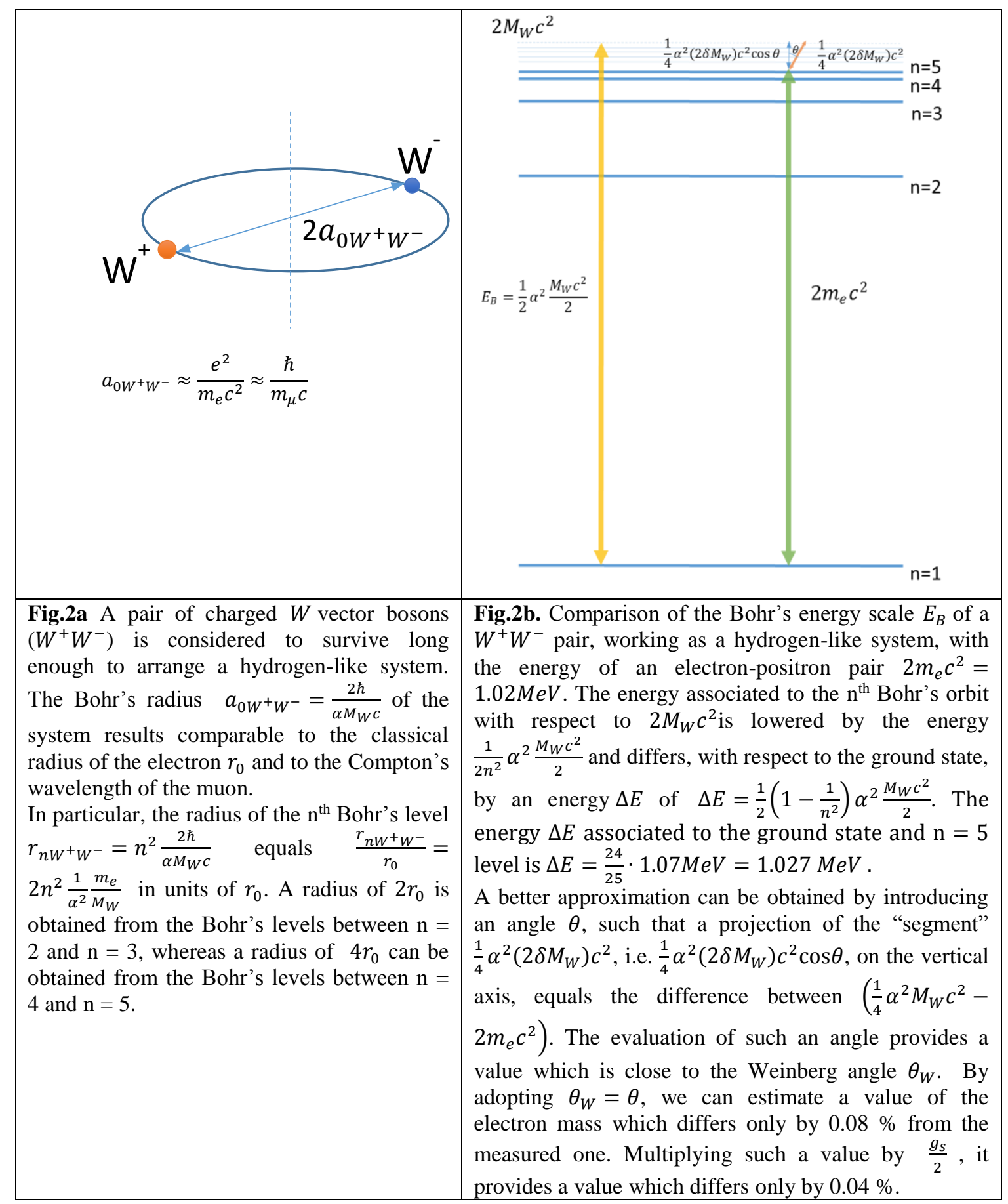




\section{References}

[1] Nakamura, K. et al. Review of Particle Physics. Journal of Physics G: Nuclear and Particle Physics. 3786, 075021-076443 (2010).

[2] Gaillard, M. K., Grannis, P. D. \& Sciulli, F. J. The standard model of particle physics. Rev. Mod. Phys. 71, 96-111 (1999).

[3] Sze, S. M. \& Kwok K. N. Physics of Semiconductor Devices (3rd ed) (John Wiley \& Sons, 2006).

[4] Cramer, C. J. Essentials of Computational Chemistry 153-189 (John Wiley \& Sons, 2002).

[5] Pavarini, E., Koch, E., van den Brink, J., Sawatzky, G. (Eds). Quantum materials: experiments and theory. Lecture notes of the autumn school on correlated electrons, https://www.cond-mat.de/events/correl16/manuscripts/correl16.pdf (2016).

[6] Kohn, W. Nobel Lecture: Electronic structure of matter - wave functions and density functionals. Rev. Mod. Phys. 71, 1253-1266 (1999).

[7] Jones, R. O. Density functional theory: Its origins, rise to prominence, and future. Rev. Mod. Phys. 87, 897- 923 (2015).

[8] Hohenberg, P. \& Kohn, W. Inhomogeneous Electron Gas. Phys. Rev. B. 136, 864-871 (1964).

[9] Kohn, W. \& Sham, L. J. Self-consistent equations including exchange and correlation effects. Phys. Rev. 140, A1133-A1138 (1965).

[10] Sasaki, S. Theory of the Integer \& Fractional Quantum Hall Effects (Nova Science Publishers, 2016). Preprint at https://arxiv.org/abs/1603.08625 (2016).

[11] Hasnip, P. J. et al. Density functional theory in the solid state. Philos. Trans. A. Mat. Phys. Eng. Sci. 372, 20130270 (2014).

[12] Weinberg, S. The Quantum Theory of Fields, Vol. I (Foundations) (Cambridge University Press, 1995).

[13] Greiner, W. \& Reinhardt, J. Quantum Electrodynamics (Springer-Verlag, 2009). 
[14] Thomson, J. J. Cathode rays. Philosophical Magazine. 44, 293-316 (1897).

[15] Wilczek, F. The enigmatic electron. Nature. 498, 31-32 (2013).

[16] Castelvecchi, D. The Strange topology that is reshaping physics. Nature. 547, 272-274 (2017).

[17] Haldane, F. D. M., Model for a Quantum Hall effect without Landau levels: condensedmatter realization of the "parity anomaly". Phys. Rev. Lett. 61, 2015-2018 (1988).

[18] Kosterlitz, J. M. \& Thouless, D. J. Ordering, metastability and phase transitions in twodimensional systems. Journal of Physics C: Solid State Physics. 6, 1181-1203 (1973).

[19] Bhattacharjee, S.M. Use of Topology in physical problems in Topology and condensed matter physics. Texts and readings in physical sciences, vol 19 (eds Bhattacharjee, S., Mj, M., Bandyopadhyay, A.) (Springer, 2017).

[20] Nash, C. Topology and Physics a historical essay in History of topology (ed. James, I. M.) 359-415 (North Holland, 1999).

[21] Morandi, G. The role of topology in classical and quantum physics in Lecture notes in physics, m7 (Springer-Verlag, 1992).

[22] Nakahara, M. Geometry, Topology and Physics (CRC Press Taylor \& Francis group, 2016).

[23] Stanescu, T. D. Introduction to Topological Quantum Matter \& Quantum Computation (CRC Press Taylor \& Francis group, 2017).

[24] Dirac, P. A. M. Quantised singularities in the electromagnetic field. Proc. Roy. Soc. A. 133, 60-72 (1931).

[25] Shnir, Y. M. Magnetic Monopoles (Springer, 2005).

[26] Aharonov, Y. \& Bohm, D. Significance of electromagnetic potentials in quantum theory. Phys. Rev. 115, 485-491 (1959).

[27] Berry, M. V. Quantal phase factors accompanying adiabatic changes. Proc. R. Soc. Lond. A. 392, $45-57$ (1984).

[28] Euler, L. Commentarii Academiae Petropolitanae 8, 128-140 (1736).

[29] Sauvage, J. P. et al. From chemical topology to molecular machines. Comptes Rendus 
Chimie. 13, 315-328 (2010).

[30] Chen, G., Wang, X., Li, X. Fundamentals of Complex Networks: Models, Structures and Dynamics (Wiley, 2015).

[31] Parks, R. D. Quantized magnetic flux in superconductors. Science New Series. 146, 14291435 (1964).

[32] London, F. Superfluids (John Wiley and Sons, 1950).

[33] Onsager, L. Proceedings of the International Conference on Theoretical Physics, Kyoto \& Tokyo. September 1953 (Science Council of Japan Tokyo, 1954).

[34] Deaver, B. S. \& Fairbank, W. M. Experimental evidence for quantized flux in superconducting cylinders. Phys. Rev. Lett. 7, 43-46 (1961).

[35] Doll, R. \& Näbauer, M. Experimental proof of magnetic flux quantization in a superconducting ring. Phys. Rev. Lett. 7, 51-52 (1961).

[36] Laughlin, R. B. Quantized Hall conductivity in two dimensions. Phys. Rev. B. 23, 5632 5633 (1981).

[37] Avron, J., Osadchy, D., Seiler, R. A topological look at the quantum Hall effect. Physics Today. 56, 38-42 (2003).

[38] Jehle, H. The relationship of flux quantization to charge quantization and the fine structure constant. Int. Journal of Quantum Chemistry. IIS, 373-375 (1968).

[39] Jehle, H. The relationship of flux quantization to charge quantization and the fine structure constant. Int. Journal of Quantum Chemistry. III, 269-287 (1969).

[40] Jehle, H. Relationship of flux quantization to charge quantization and the electromagnetic coupling constant. Phys. Rev. D. 3, 306-345 (1971).

[41] Jehle, H. Flux quantization and particle physics. Phys. Rev. D. 6, 441-457 (1972).

[42] Jehle, H. Flux quantization and fractional charges of quarks. Phys. Rev. D. 11, 2147-2177 (1975).

[43] Jehle, H. Topological characterization of leptons, quarks and hadrons. Physics Letters B. 104, 207-211 (1981). 
[44] Saglam, M., Boyacioglu, B.,Saglam, Z., Yilmaz, O. \& Wan, K. K. Spin-dependent quantized magnetic flux through the electronic orbits of Dirac hydrogen atom. Preprint at https://arxiv.org/ftp/physics/papers/0608/0608165.pdf (2006).

[45] Stein, W. D. R. Quantized magnetic flux through the orbits of hydrogen-like atoms within the atomic model of Sommerfeld. Preprint at https://arxiv.org/pdf/1302.6163v1.pdf (2013).

[46] Wilson, K. G. Confinement of quarks. Phys. Rev. D. 10, 2445-2459 (1974).

[47] Pohl, R. et al. The size of the proton. Nature. 466, 213-216 (2010)

[48] Jackson, J. D. Classical Electrodynamics (3rd ed.) (John Wiley \& Sons, 1999).

[49] Barut, A. O. The mass of the muon. Phys. Lett. B. 73, 310-312 (1978).

[50] Beringer, J. et al. Review of particle physics. Phys. Rev. D. 86, 010001 (2012). 